\title{
THE WONDERFUL WORLD OF GOLF: A PHILOSOPHICAL TREATMENT OF THE SIMILARITIES THAT EXIST IN GOLF AND ANAESTHESIA*
}

\author{
ERUC WeBb, M.D. $†$
}

My APPROACH to teaching anaesthesia emphasizes the use of simple analogies. Golf, an increasingly popular game (and business), is a particularly fruitful source of situations that can be applied to anaesthesia. I claim no originality in this. Anesthesiology carried an editorial entitled "Golf and Anesthesia" in its 1942 columns.

Golf is primarily a game, played for pleasure. As soon as you play golf professionally, you realize the need for perfection-not proficiency but perfection! The quest for perfection creates tension. Unless the tension can be controlled and channelled, disaster rather than perfection results. This is my first analogy. Unless the concern for perfection in anaesthesia is present, and unless the tensions contribute to perfection more than they disturb function, the anaesthetist will score many bogies.

\section{Get Me to the Tee on Time}

Probably the worst preparation for a game of golf is to arrive at the clubhouse forty minutes late, having rushed away from a case that has gone long past your estimated time, and having left the patient in rather poor condition in the recovery room. You inhale a sandwich and a beer as you dress, then rush, apologetic and apoplectic, to the first tee. Contrast this to the round on your holidays -rested, well fed, relaxed, and punctual. Likewise, in the operating theatre, things go much better if the anaesthetist can arrive ahead of time, prepare equipment and drugs in a leisurely, relaxed fashion, and be ready to begin his work at the scheduled hour.

\section{Carry a Full Set of Clubs}

The golf duffer may not be impressed with the need for a full set of clubs, but the professional always takes advantage of the PGA rule that allows fourteen clubs in the bag. Indeed, depending on the course, weather, wind direction, and other considerations, the fourteenth club may differ from match to match.

Every club, properly stroked, yields a predictable result. It is possible to play a round using the four iron for every shot. Indeed some trick shot artists make this into a golfing side show. The player who carefully selects his clubs will on the whole score much better.

There are at least four directions that this discussion may take.

'Presented at the Western Division Meeting, Canadian Anaesthetists' Society, Vancouver, B.C., May 29, 1968.

tDepartment of Anaesthesia, Vancouver General Hospital, and the Faculty of Medicine, University of British Columbia. 


\section{Relaxants}

The relaxant drug that most closely meets the need of the anaesthetic situation is the one that should be administered. Giving repeated small doses of succinylcholine is comparable to hitting the ball eight or nine times with a putter, instead of once with a driver. Decamethonium acts for a predictable fifteen to twenty minutes, and will provide excellent conditions in the case where this is what is required. The often-seen attempts to apply the same relaxant to every situation handicaps patient, anaesthetist, and surgeon.

\section{Opiates}

Opiate drugs have more or less predictable differences, and it irks me that in spite of these predictable differences a single drug, say alphaprodine, will be flogged to death, merely because of a short duration. What of the case where the short duration of action is a nuisance, requiring repeated injections? Occasionally, is not the use of a sizeable bolus of an opiate better than creeping up on the patient with a dilute drip?

\section{Laryngoscope blades}

If the medium-sized Macintosh blade is the only one available, there is scant virtue in claiming "I always use the medium-sized Macintosh for every case," a remark that has earned residents at our hospital more than one unkindly rebuke. I carry two Macintosh blades, small and medium; two Guedels, small and medium; a Bennett medium; a (self) modified Miller; and an Oxford Guedel for infants. With this assortment, I can, I firmly believe, make an intelligent choice of the blade that will make visualization of the vocal cords easiest. Where the patient has lost his upper teeth, I go to the Guedel first. I try to differentiate between the toothless infant, the 2\%-year-old, and the four-year-old, and fit the blade to the throat as it were. My Bennett blade is used maybe once a year, and it may be by an associate who borrows it knowing it will help. If I am experiencing difficulty with an intubation sometimes a switch in blades turns an almost impossible intubation into a very easy one. And, while we are discussing laryngoscope blades, blind nasal intubation should be practised at every available opportunity just to keep one's hand in.

\section{Anaesthetic techniques}

This is probably the best facet of anaesthesia to apply the "full set of clubs" philosophy.

One unforgettable Sunday evening about 1960, on his first operating room contact with me, a new resident suggested, as his technique of choice for three cases in a row, "Anectine drip and nitrous." As the three cases were respectively a D \& C, an appendicectomy in an athletic 23-year-old male, and a supracondylar fracture of the humerus in an eight-year-old girl, I was a trifle concerned. My personal preferences would have had his choice well down on my list. I asked if he ever used any other technique of anaesthesia. (That at least was the gist of what I asked him.) In a year of so-called training in another hospital he had never seen or been shown any other way of maintaining anaesthesia. Having just 
returned from a visit south of the border, and having seen four well-established anaesthetists do the identical type of practice, adding mechanical ventilation, my conclusion is that such "cookbook" attitudes are a disgrace to our specialty. This is sad and even wicked when it is the result of judicial pressures. Where some of the department's students are aiming towards a specialist status, and others are heading for isolated practices, a variety of technical approaches is still more mandatory. We should insist that anaesthetic attitudes do not become stereotyped and circumscribed; we should keep ourselves and our attitudes sharp and precise.

\section{Slow Golfers Are a Nuisance}

By and large, the five-hour round of golf is as soul destroying as the five-hour game of professional baseball. After one putt that had been lined up again and again over some six or seven minutes had failed to drop, in fact it had not really come close to dropping, the impatient opponent growled, "You could have been twice as close in half the time." Many surgeons could, in all fairness, make this retort to many anaesthetists.

Show-off tactics and speed for speed's sake are unforgivable in any medical effort, but I deplore the time wasted by unorganized or disorganized, compulsive, non-contributory manoeuvres by the anaesthetist. I admit that there are occasions -such as when $\$ 25,000$ hang on a long putt-that suggest a careful and steady assessment before precipitous action. A deliberate and gentle induction of a high risk patient may pay good dividends. But slow anaesthesia as a first general principle is neither necessary nor desirable.

\section{Please Replace Your Divots}

A good golfer will take a divot every stroke if necessary, but on a well-organized course these divots are replaced and the general well-being of the fairways is not disturbed. After a shot out of a bunker, the polite golfer smoothes the sand for the next player. One of my special side interests is anaesthetic equipment, and I hate to see equipment abused or neglected. Early in my training, I was taught to rinse blood from syringes as soon as possible. It should not be beneath the anaesthetist's dignity to submit his recently removed endotracheal tube to a rinse under the tap. Small ancillary equipment should be carefully used, and valve assemblies, canisters, and the like should be the anaesthetist's care, not left entirely to the departmental technicians.

\section{Stay Loose}

Be adaptable, in other words. Golf in the tropics is very different to golf in Vancouver. The humid heat makes an eighteen hole effort almost impossible. Tarred or oiled sand putting surfaces require a new technique. The grass grows very quickly in the wet season, and in the dry you are shooting from a sandy surface nearly all the time. Approaches don't catch. When I played there the crowded membership demanded all sorts of (to us) queer starting regulations, and almost every day there were different playing regulations; no four balls on one day; three balls no standing certain days. It called for adaptability. This is true of anaesthetists in the theatre every day. Don't be rigid. 
On my first official visit to the Orthopaedic Hospital in Lagos the first case was a wee one-year-old for sequestrectomy of the femur. When I asked for the infant equipment, I was shown a bottle of ethyl chloride (which I had not used since 1944), a Pentothal bottle with Pentothal dispenser, full of ether, a couple of small masks, and a couple of small airways. Since this was the first demonstration of the new Canadian Expert's ability, the case had to go smoothly and it did. But a great deal of that tension I was discussing earlier was in my mind, even if the surgeon might have had a hard time diagnosing it.

\section{Go for Broke}

Every golfer, duffer or professional, has been faced with the "go for broke" problem. The proper tactical approach to one of these chance-taking shots may differ greatly depending on the precise circumstances. Is it early in the game or late? Are you ahead comfortably, or trailing miserably if the shot doesn't come off? Do you need it to win, or to tie? However, on the golf course, a chance taken and missed loses a hole, a match, at most a title or a sizeable amount of money. In the operating room the surgeon says "I know we"ve no blood but I think I'll take a chance." What chance is he taking? The patient is the one at risk. Always remember this, and treat the patient's risk as if it were your own.

\section{The Nineteenth Hole}

The nineteenth hole has become, or maybe always was, the focal point of the whole game of golf. Overdone, if it develops into twenty-six or seven, it may be less than wholly desirable. But the friendly relaxation of the after-game drink is an essential and wonderful part of Thursday afternoon. I do not feel that the nineteenth hole in our anaesthetic analogy is the friendly cup of coffee with the members of the surgical team. It is rather a quiet moment or two, more if indicated, with the patient in the recovery room, making sure that things are going well with him. After you assure yourself of that, then is the time to slip along to the twentieth hole, your coffee with your pals.

We have by no means exhausted the possibilities of this discussion, but I think I have made a few points from the fairway to the operating table. Just as the golfer strives to lower his score-the hundred shooter wants to break 100, the low ninety man wants to get into the eighties, and the professional is unhappy unless he can card four rounds in a row, all sub par-so the anaesthetist should have in his mind a series of cases, all of which have been "par or sub par rounds." The green anaesthetist wants to do better. The specialist wants all his cases if possible to be examples of perfection. 